Projets

de paysage

\section{Projets de paysage}

Revue scientifique sur la conception et l'aménagement de l'espace

$8 \mid 2012$

Les concepteurs de jardins et de parcs japonais

\title{
Le développement périurbain du Pays de France : des influences urbaines différenciées
}

The peri-urban development of the "Pays de France" : different urban influences

\author{
Jean-Baptiste Grison
}

\section{OpenEdition}

\section{Journals}

Édition électronique

URL : http://journals.openedition.org/paysage/14838

DOI : $10.4000 /$ paysage. 14838

ISSN : 1969-6124

\section{Éditeur :}

École nationale supérieure du paysage de Versailles-Marseille, Institut national des sciences appliquées Centre Val de Loire - École de la nature et du paysage, École nationale supérieure d'architecture et de paysage de Bordeaux, École nationale supérieure d'architecture et de paysage de Lille, Agrocampus Angers

Référence électronique

Jean-Baptiste Grison, « Le développement périurbain du Pays de France : des influences urbaines différenciées », Projets de paysage [En ligne], 8 | 2012, mis en ligne le 12 octobre 2012, consulté le 11 mars 2021. URL : http://journals.openedition.org/paysage/14838 ; DOI : https://doi.org/10.4000/ paysage. 14838

Ce document a été généré automatiquement le 11 mars 2021.

Projets de paysage 


\title{
Le développement périurbain du Pays de France : des influences urbaines différenciées
}

The peri-urban development of the "Pays de France" : different urban influences

\author{
Jean-Baptiste Grison
}

1 Au nord de l'aire urbaine de Paris, le Pays de France peut être assimilé à un espace encore en partie rural, entrecoupé de quelques petites villes, et englobé au sein de la couronne périurbaine de Paris, dans laquelle il forme une sorte de " poche » au paysage encore partiellement agricole, encadré par des axes où la progression urbaine a été plus importante ${ }^{1}$.

Dans ce secteur, la croissance de l'espace bâti est une réalité constante depuis les années 1920. Les différentes phases de ce développement sont bien lisibles dans le paysage. Une des problématiques majeures de cet espace est d'être encadré par des ensembles urbains aux contenus divers, en rupture les uns avec les autres, d'un point de vue sociospatial (figure 1 ):

- au sud, la banlieue nord de l'agglomération parisienne est caractérisée par une forte surreprésentation de l'habitat populaire, liée à la fois à un héritage industriel notoire, et à un paysage résidentiel fortement marqué par les lotissements ouvriers de la première moitié $\mathrm{du} \mathrm{xx}^{\mathrm{e}}$ siècle, puis par les grands ensembles des années 1960 et 1970 ;

- à l'inverse, au nord, l'agglomération de Chantilly accueille, depuis plusieurs décennies, des populations aux revenus élevés, dans des lotissements et des résidences de bon standing. Ces caractéristiques se retrouvent, bien qu'un peu atténuées, au nord-ouest de la zone, autour des forêts de Carnelle et de l'Isle-Adam, qui représentent, à l'instar de celle de Chantilly, des aménités paysagères évidentes ;

- enfin, au sud-est, la plateforme aéroportuaire de Roissy, développée depuis les années 1970, constitue un immense pôle d'emplois (le plus important du nord de l'île-de-France), présentant une palette de métiers et de qualifications très variée. 
3 Le paysage du Pays de France, en dehors des espaces bâtis, est avant tout celui d'un plateau agricole où les grandes cultures dominent largement. Forêts et prairies sont cantonnées à quelques vallons, ainsi qu'au fond de la vallée de l'Ysieux qui le traverse d'est en ouest. Plusieurs buttes témoins, sur lesquelles des villages se sont installés (Mareil-en-France, Châtenay-en-France, Saint-Witz) offrent de larges panoramas sur l'agglomération parisienne ou le Sud de la Picardie. À l'orée de l'avènement des phases successives de développement urbain et périurbain (fin du XIX ${ }^{\mathrm{e}}$ siècle), l'habitat était réparti en villages groupés et de taille le plus souvent modeste, de la centaine au millier d'habitants.

4 À partir de ces constats, notre hypothèse est que le développement périurbain du Pays de France subit les influences croisées des espaces qui l'entourent. Comment leurs caractéristiques s'imbriquent-elles dans les différentes localités ? Y a-t-il des clivages spatiaux internes à cet espace périurbain, ou celui-ci prend-il la forme d'une transition progressive entre l'agglomération parisienne, la plateforme aéroportuaire et l'aire cantilienne?

5 Nous proposons, dans cet article, de relire ce développement en analysant les dynamiques inhérentes à ces trois influences externes différentes (bien qu'étroitement liées) agissant de manière différente, voire contradictoire, sur le Pays de France et la morphologie paysagère et sociale de son espace bâti.

Figure 1. Le Pays de France, un territorie périurbain encadré par l'urbain

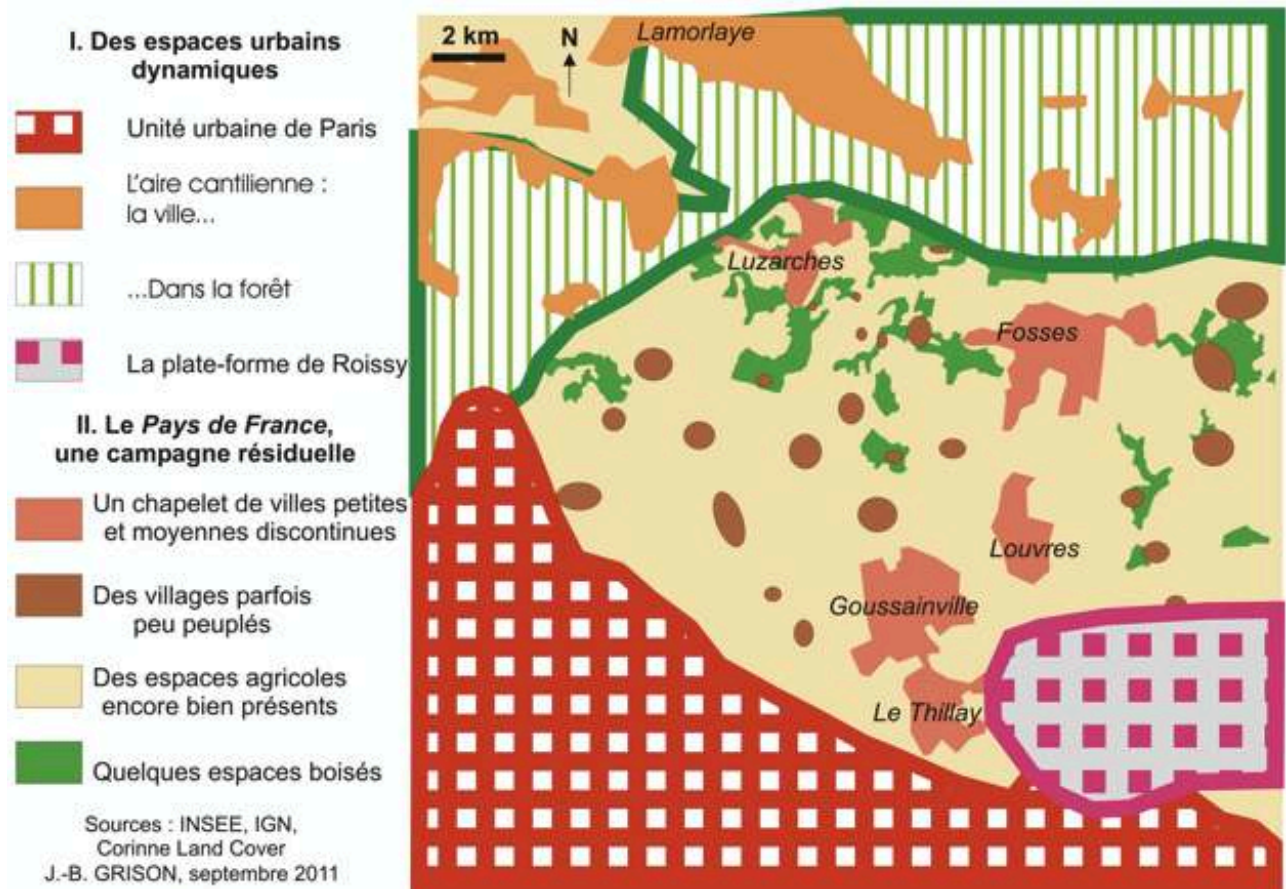

\section{La dynamique métropolitaine et polarisante de l'agglomération parisienne : une logique radiale}

6 La masse compacte de l'agglomération parisienne forme une couronne, bien visible sur la carte, allant jusqu'à une vingtaine de kilomètres du centre de la capitale. Au-delà de 
cette urbanisation continue, le développement de l'espace bâti se poursuit le long d'axes radiaux obéissant à des logiques de plusieurs formes (figure 2). Par ailleurs, le nord de l'agglomération parisienne est globalement caractérisé par une prédominance de quartiers traditionnellement occupés par des catégories sociales plutôt populaires. Au-delà du bâti continu de l'agglomération, une partie de l'extension périurbaine répond à cette pression populaire.

\section{Une urbanisation en chapelet à partir des gares ferroviaires}

7 Le Pays de France est traversé par la voie ferrée Paris-Creil (ligne classique Paris-Lille). Historiquement, celle-ci fut le principal vecteur de développement périurbain dans ce territoire (Clozier, 1940). Les principales gares le desservant sont Goussainville, Louvres et Survilliers-Fosses. En général, l'urbanisation s'est faite directement à partir des gares, indépendamment de la localisation du village historique situé quelquefois à plus de trois kilomètres. Dans le cas de Fosses et de Puiseux-en-France (agglomération de Louvres), il y a toujours un espace non bâti entre la ville actuelle et le village d'origine.

8 À partir de cette desserte ferroviaire, le développement s'est fait, dans un premier temps, principalement sous la forme de lotissements de type ouvrier, densifiés au fil des décennies à partir des années 1920. Les localités de Goussainville, de Louvres, de Puiseux-en-France, de Fosses, de Marly-la-Ville et de Survilliers ont progressivement formé un chapelet de petites villes, qui a pris définitivement cette forme à partir des années 1950.

Dans les années 1960 et 1970, la progression urbaine a changé de nature, au profit du développement d'ensembles d'immeubles et de lotissements dans les petites villes déjà formées par l'urbanisation autour des gares. On aboutit ainsi à une densification de cette progression urbaine sur l'axe de la voie ferrée, dans la mesure où cette génération de constructions a été souvent l'occasion d'un bond important dans la taille des localités concernées. Si Goussainville et Le Thillay s'étaient un peu plus développés au cours des décennies précédentes, les communes de Louvres, de Puiseux-en-France, de Fosses et de Survilliers ont plus que doublé leur parc de logements au cours de la seule période intercensitaire 1968-1975 (respectivement 127, 160, 176 et $125 \%$ d'augmentation). Il en fut de même, entre 1975 et 1982, pour Marly-la-Ville (+108 \%) et Saint-Witz (+ $551 \%$ !). Enfin, depuis les années 1990, un mouvement de requalification et densification des quartiers de gare est observé.

Aujourd'hui, ces ensembles prennent des formes nettement plus urbaines, et sont clairement qualifiables de villes-dortoirs de la banlieue parisienne, tout en restant morphologiquement discontinues. Situées sur le plateau, construites au hasard des opportunités foncières dégagées sur le passage du chemin de fer, ces localités sont apparues en rupture nette avec l'environnement paysager préexistant, ignorant les sites plus déterministes des villages ancestraux. Si les lotissements ouvriers, densifiés très progressivement depuis les années 1920 , sont aujourd'hui très hétéroclites, les opérations immobilières des années 1960 ou 1970, qui ont développé l'habitat collectif ou individuel, laissent en héritage de vastes quartiers résidentiels très homogènes.

11 La discontinuité de ces espaces à dominante urbaine donne cependant aux paysages qui y sont associés des relents de ruralité, qui se traduisent encore dans une partie des politiques locales, comme le fait valoir, par exemple, le slogan de la municipalité de Fosses : «La ville à la campagne.» Ainsi, dans les anciens lotissements ouvriers, 
quelques éléments de basse-cour ne sont pas rares, tandis que depuis les fenêtres des immeubles de logements sociaux, l'agriculture est presque toujours présente dans le paysage. En outre, l'espace agricole enveloppant ces petites villes, même s'il se réduit d'année en année, bénéficie d'un entretien satisfaisant, malgré des mutations manifestes, notamment en faveur des jardins ouvriers, d'une part, et des pépinières, d'autre part. La plupart des communes comptent encore au moins une exploitation professionnelle.

Figure 2. L'influence de l'agglomération parisienne : une logique radiale

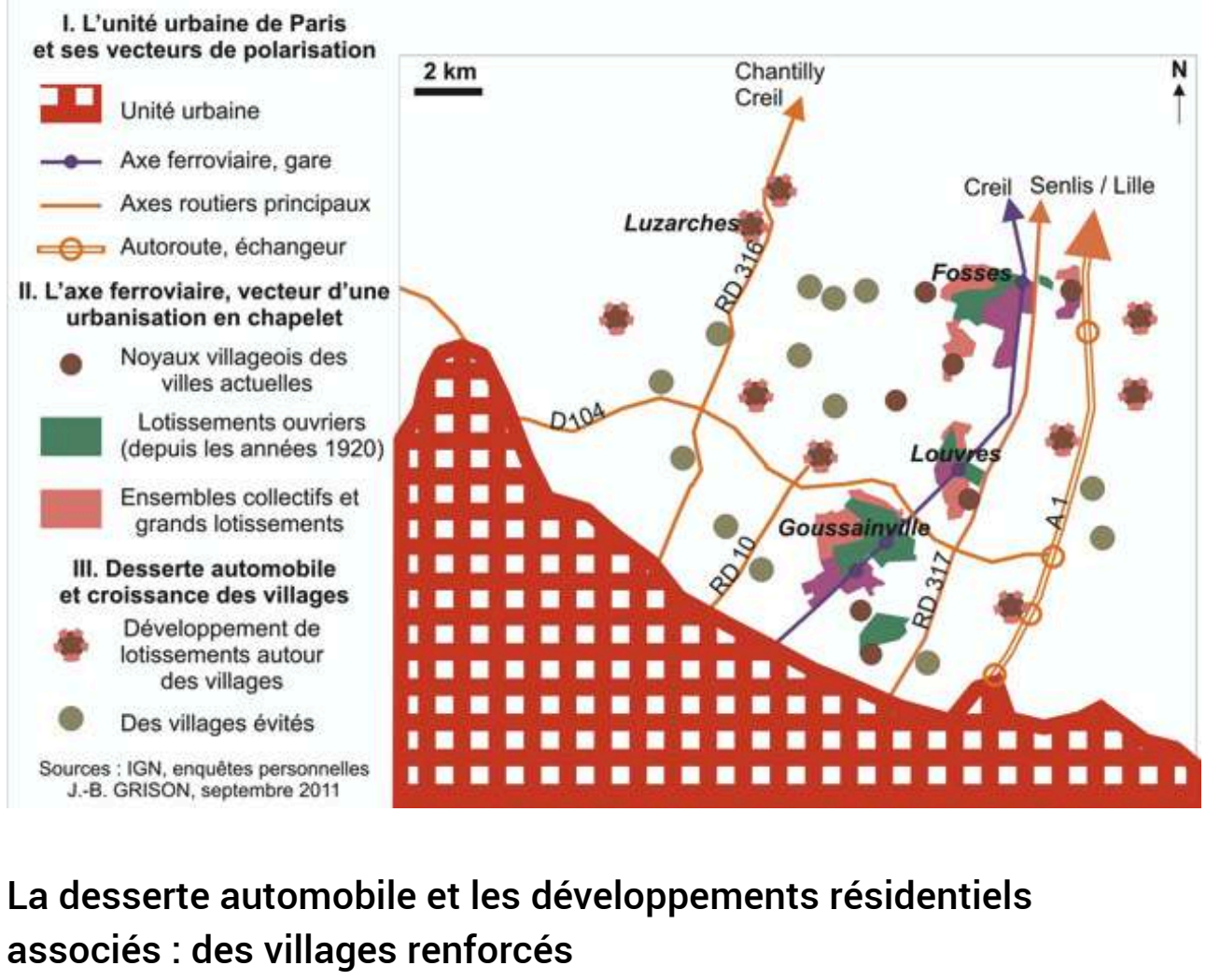

Le Pays de France est traversé par trois radiales majeures : les routes départementales 316 et 317 (anciennes nationales 16 et 17), ainsi que l'autoroute A1. Entre les années 1960 et les années 1990, une phase de développement a entraîné la croissance de quelques villages sur la base d'une desserte davantage routière que ferroviaire. Des lotissements ont conduit plusieurs vieux villages facilement accessibles en voiture (parfois très peu peuplé historiquement) vers un volume démographique à la limite du gros bourg et de la petite ville (au sens de l'Insee ${ }^{2}$, bien que le paysage soit en général typiquement celui de gros villages périurbains). Il s'agit de Saint-Witz, Vémars, Fontenay-en-Parisis, Villeron, pour l'essentiel.

Ce développement correspond à un mouvement très banal de la périurbanisation. Dans le paysage, les lotissements correspondants sont peu individualisés, mais les centres villageois ancestraux sont désormais noyés dans une large couronne de pavillons contemporains très standardisés, progressant sur le plateau. Cependant, on note quelques différences, d'un village à l'autre, ou plutôt d'un quartier à l'autre, dans le standing des quartiers et la nature des populations attirées. Par exemple, le village de Saint-Witz s'est développé principalement dans la perspective d'attirer des ménages à 
moyens ou hauts revenus, tandis que Vémars ou Fontenay-en-Parisis ont mené des opérations plus contrastées. Par ailleurs, tous les villages situés le long des axes radiaux n'ont pas suivi ce modèle de développement. Par exemple, Le Mesnil-Aubry, le long de l'ancienne nationale 16, a maintenu une certaine stabilité durant toute la seconde moitié $\mathrm{du} \mathrm{xx}^{\mathrm{e}}$ siècle, malgré sa bonne desserte routière et sa proximité de Paris. Globalement, le cœur du Pays de France a plutôt résisté à cette forme d'urbanisation.

La croissance périurbaine directement influencée par la banlieue nord de l'agglomération parisienne obéit bien à une logique radiale, mais discontinue (ce qui est le propre d'un espace périurbain), s'appuyant sur des points de fixation: les gares, les échangeurs autoroutiers, ou les noyaux villageois situés à proximité des grands axes. La périurbanisation qui en résulte peut être qualifiée de "quantitative » ou "de masse ", dans la mesure où elle se traduit par des opérations immobilières d'ampleur significative, tendant vers une morphologie résidentielle aux caractéristiques clairement urbaines. Par ailleurs, la plupart des grandes phases qui ont conduit la croissance de l'aire urbaine de la capitale se retrouvent dans ce secteur (Bastié, 1984). Cependant, le fait que tous les villages ne soient pas concernés, et qu'un véritable gradient de développement périurbain à partir de la capitale ne puisse être vérifié, prouve qu'il existe des formes de résistance à cette croissance, répondant à d'autres logiques.

\section{La dynamique environnementale et récréative de l'aire cantilienne : une logique frontale}

Bien qu'étroitement lié à l'agglomération parisienne, le développement de l'aire cantilienne (forêt de Chantilly et ses abords), remontant, lui aussi, au début du $\mathrm{xx}^{\mathrm{e}}$ siècle, a son influence propre sur le Pays de France, essentiellement sur sa partie nord. Au Sud de la Picardie, la région de Chantilly correspond à une entité paysagère assez différente de celle que nous étudions ici, constituée d'une alternance de vastes forêts (Chantilly, Ermenonville, Carnelle), et d'espaces urbanisés, les communes concernées comptant, en règle générale, quelques milliers d'habitants.

\section{Des particularités résidentielles}

16 Les premiers développements résidentiels associés à la forêt de Chantilly, demeurant aujourd'hui les plus spectaculaires, sont ceux du Lys (communes de Lamorlaye et de Gouvieux), juste au nord du Pays de France. Ce vaste domaine de 700 hectares, privé, comportant 1640 parcelles boisées, a été loti à partir de 1925 sur une ancienne propriété ducale, visant dès le départ une population aisée en provenance, principalement, de l'agglomération parisienne. Cette première réalisation de grande ampleur a suivi le mouvement, observé depuis le xixe siècle, de construction (ou réaménagement) de villégiatures bourgeoises dans les localités alentour (Bastié, 1984). Par la suite, l'unité urbaine de Chantilly a accueilli d'autres opérations immobilières visant le même type de clientèle, à une échelle plus restreinte. Enfin, dans le courant des dernières décennies, le patrimoine des anciens noyaux villageois a également été réinvesti par des ménages aux revenus relativement confortables, et généralement très liés à la région capitale (bien que Chantilly se trouve, administrativement, en Picardie). La rapidité de la liaison ferroviaire Chantilly-Paris (une vingtaine de minutes) et la 
proximité du pôle d'emploi de Roissy semblent jouer un rôle majeur dans cette attractivité (Faburel et Barraqué, 2002), et s'ajoutent aux aménités paysagères.

$\mathrm{Au} \mathrm{vu}$ de ces quelques caractéristiques élémentaires, il est évident que cette aire cantilienne présente un visage radicalement différent, voire opposé sur bien des points, à celui de la banlieue nord de Paris. Il est tout aussi évident qu'elle exerce, elle aussi, une certaine influence sur l'évolution du Pays de France, avec lequel elle voisine au sud. Ainsi, Luzarches et Chaumontel, deux communes formant une petite agglomération urbaine, répondent assez bien à la morphologie des petites villes du sud de l'Oise, dans un cadre paysager préservé. Les hameaux alentour ont accueilli au fil des décennies un nombre significatif de belles demeures, isolées ou en petits lotissements. Il en est de même, un peu plus loin, pour les petites communes d'Épinay-Champlâtreux et de Châtenay-en-France, qui, malgré une bonne desserte routière, n'ont jamais libéré d'emprise foncière au profit de lotissements pavillonnaires, mais leurs belles demeures nobiliaires ou bourgeoises sont restées bien mises en valeur. À Mareil-en-France, le hameau des Fabriques est un petit ensemble de villas luxueuses construites au début du $\mathrm{xx}^{\mathrm{e}}$ siècle, accompagnées d'un parc et de quelques équipements récréatifs communs. Enfin, des villages comme Jagny-sous-Bois, Lassy, Le Plessis-Luzarches, ont maintenu jusqu'à ce jour leur périmètre initial, et leurs constructions neuves, peu nombreuses, sont soumises à des cahiers des charges stricts (parfois propres à un petit lotissement, mais de plus en plus souvent généralisés dans le document d'urbanisme municipal) répondant à une réelle volonté de valorisation paysagère de l'espace résidentiel. Dans cette partie du Pays de France, l'habitat se valorise principalement dans des sites paysagers évitant la monotonie du plateau et profitant des irrégularités du relief: vallons reculés (hameau d'Hérivaux, Jagny-sous-Bois), vallée de l'Ysieux (Le PlessisLuzarches, Lassy, hameau de Thimécourt), lisières de bois (Épinay-Champlâtreux), buttes témoins (Mareil-en-France, Châtenay-en-France). À l'instar de l'aire cantilienne, la valorisation résidentielle s'appuie en premier lieu sur les aménités paysagères.

\section{Une dynamique environnementale et de protection de l'espace}

18 Au-delà de ces caractéristiques résidentielles, destinées à une population plutôt aisée et donc radicalement différente de celle résultant de la logique précédente, l'aire cantilienne a été assez tôt concernée par la mise en place de dispositifs de protection de l'espace, à commencer par le château et l'important domaine forestier d'État qui l'entoure. Le lotissement du Lys, dès sa création, a fait l'objet d'un règlement draconien visant à préserver son authenticité. La totalité des forêts de la zone, ainsi qu'une grande part du périmètre urbanisé sont aujourd'hui classées sous le régime de la loi du 2 mai 1930 (sites classés ou inscrits).

Dans cette logique, l'influence de l'aire cantilienne sur le Pays de France se caractérise aussi par une volonté croissante de protéger l'espace. Aujourd'hui, l'essentiel de la partie rurale de ce territoire est protégé, les périmètres s'étant progressivement étendus: le domaine de Chantilly, classé en 1960, déborde de quelques centaines d'hectares sur les communes de Luzarches et Chaumontel; l'ancienne abbaye d'Hérivaux et ses alentours ont été classés en 1982; la butte de Châtenay en 1989; enfin, le classement des vallées de l'Ysieux et de la Thève, en 2002, a permis de couvrir une zone plus vaste, étendue sur treize communes et un peu plus de 4000 hectares, pour sa partie située dans le département du Val-d'Oise (figure 3). 
torze communes du Val-d'Oise ont en outre rejoint le Parc naturel régional OisePays-de-France, créé en 2004. Cette dynamique de préservation accentue sensiblement l'effet de "front ", clairement observable, tant sur les cartes que dans le paysage, face à l'agglomération parisienne compacte. Ce dernier périmètre traduit aussi la volonté d'identifier cette partie du territoire à l'aire cantilienne et au massif des Trois Forêts (Chantilly, Halatte, Ermenonville). Malgré l'existence d'une polarisation évidente de tout cet espace vers Paris, le souhait de mettre en avant une image plus indépendante et moins directement parisienne est manifeste.

\section{Une bonne place des loisirs récréatifs dans le paysage et l'identité territoriale}

La forêt de Chantilly est l'une des plus fréquentées de France. En outre, la ville est historiquement reconnue comme "capitale du cheval ", et les activités équestres y prennent une place très importante. D'autres loisirs à haute valeur ajoutée, comme le golf, s'y sont abondamment développés, répondant à la fois à la demande de populations résidentes à hauts revenus, et à celle de citadins en provenance du cœur de l'agglomération parisienne, en recherche d'aménités paysagères pour la pratique de ces loisirs.

Les installations associées aux pratiques de l'équitation et du golf, très consommatrices d'espaces, se sont développées dans une partie du Pays de France, sous l'influence directe de l'aire cantilienne. Ces installations sont essentiellement cantonnées au nordouest de la zone, dans les communes les plus proches de Chantilly: elles restent, globalement, en deçà d'un arc Fosses - Châtenay-en-France - Belloy-en-France, bien que quelques centres équestres aient vu le jour à Louvres, Saint-Witz et Vémars.

D'autres activités de loisirs profitent de ces aménités en valorisant quelques grands domaines: le château de Champlâtreux, monument historique, est loué par ses propriétaires pour des réceptions de grand standing; un centre de séminaires à Châtenay-en-France vise, lui aussi, une clientèle haut de gamme ; enfin, le domaine de Lassy, propriété du groupe Air France, offre une large palette d'activités sportives dans le cadre enchanteur du domaine d'une ancienne propriété bourgeoise de 27 hectares... 
Figure 3. L'influence de l'aire cantilienne : une logique frontale

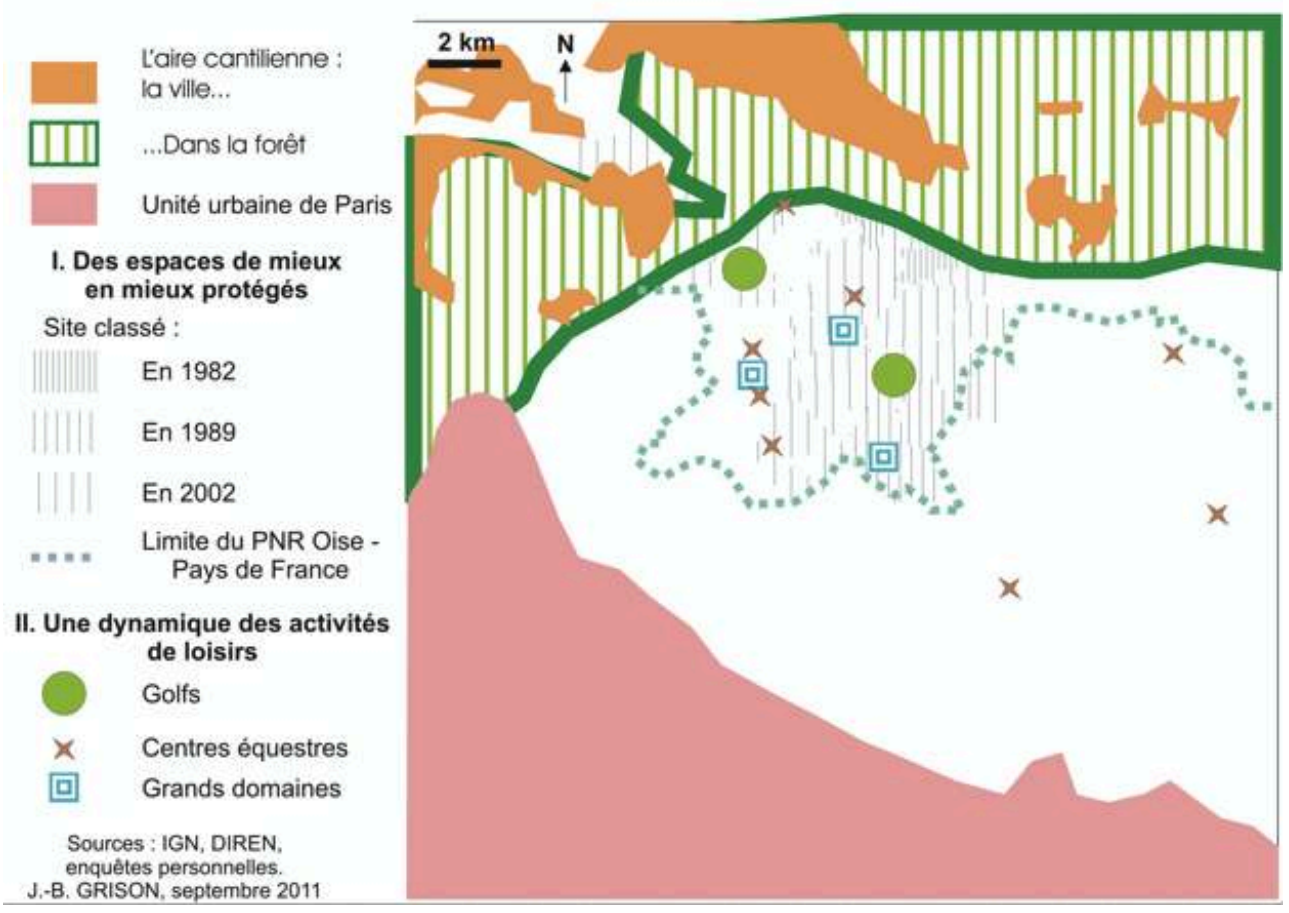

Dans l'ensemble, on peut considérer que l'influence cantilienne, sur les trois angles abordés, correspond principalement à une diffusion en tache d'huile, conduisant à la formation d'un "front" l'opposant à une banlieue nord de Paris radicalement différente. Cette logique frontale connaît une relative stabilité spatiale, mais le contraste tend à s'amplifier au fil des décennies. Les politiques d'urbanisme des dernières années se poursuivent dans ce sens.

En somme, la logique frontale de l'influence cantilienne traduit une opposition grandissante, de la part des acteurs émanant des localités au cadre " préservé ", face à des dynamiques d'urbanisation plus massive en provenance de la banlieue nord parisienne. À titre d'exemple, le contraste entre les deux communes de Goussainville et de Châtenay-en-France, distantes de seulement quelques kilomètres, est saisissant: tandis que la première a connu au fil $\mathrm{du} \mathrm{xx}^{\mathrm{e}}$ siècle un développement urbain considérable, qui en fait aujourd'hui une ville périphérique d'une trentaine de milliers d'habitants, composée d'une alternance de quartiers pavillonnaires issus des anciens lotissements ouvriers et de grands ensembles collectifs de logements sociaux, la seconde, qui n'a toléré quasiment aucune construction depuis le xix siècle, partage l'essentiel de son territoire entre quelques grandes propriétés bourgeoises, et dépasse à peine la soixantaine d'habitants. Par ailleurs, l'écart de revenu moyen des foyers fiscaux était de 1 à 4 au début des années 2000, entre les deux localités (Grison, 2008).

\section{La dynamique d'activités de la plateforme de Roissy: entre diffusion et fracture spatiale}

L'ouverture, en 1974, de l'aéroport Roissy-Charles de Gaulle a bouleversé l'organisation du Pays de France. Cet équipement de grande envergure, aujourd'hui indispensable à la région capitale, entraîne deux logiques d'organisation en plus des précédentes, l'une 
liée aux nuisances inhérentes au trafic aérien, l'autre à l'ampleur des activités économiques générées, plus ou moins directement, par la plateforme.

\section{Un couloir de bruit qui dicte l'organisation de l'espace}

27 Une des particularités de l'équipement aéroportuaire est d'engendrer, au-delà de son emprise spatiale propre, un couloir de nuisances sonores qui conduit à dévaloriser l'espace concerné. Dans le cadre de Roissy, premier aéroport français, le nombre très important de rotations engendre un niveau de bruit considérable le long d'un couloir s'étendant à l'est et surtout à l'ouest de l'emprise (en raison des vents dominants qui dictent le sens des décollages). Dans cette partie ouest, qui concerne justement le Pays de France, la bande de nuisances suit à peu près les limites de l'unité urbaine de Paris (figure 4).

En regardant de plus près les localités concernées par cette bande de nuisances, on se rend compte assez aisément $\mathrm{du}$ frein que l'aéroport a introduit dans leur développement, et de la rupture dans l'urbanisation ainsi provoquée. Épiais-lèsLouvres, Vaud'herland, le "vieux pays» de Goussainville, Bouqueval et Le PlessisGassot portent clairement les marques de cette dévalorisation (Cavard, 1994).

Ces villages ont conservé un caractère rural, non pas par souci de préservation face à une pression urbaine redoutée comme dans le cas de la protection associée à l'aire cantilienne, mais par impossibilité locale de s'étendre. On aboutit ainsi à une zone d'évitement, voire de rupture ou de fracture, dans la progression urbaine de l'agglomération parisienne. Plusieurs villages sont en effet frappés par l'interdiction de toute construction résidentielle nouvelle liée à leur localisation dans les zones A et B du plan d'exposition au bruit, qui correspond à un niveau sonore moyen supérieur à 60 décibels (Nadler, 2004).

Dans cette zone de frange urbaine où la croissance résidentielle a été ainsi " sacrifiée » aux nuisances sonores, d'autres équipements porteurs, eux aussi, de nuisances et nécessaires au fonctionnement de l'agglomération parisienne se sont également installés: il s'agit, d'une part, du poste électrique du Plessis-Gassot, imposante concentration de lignes à haute tension alimentant une partie de la région île-deFrance; d'autre part, du centre d'enfouissement du Plessis-Gassot qui s'étend sur 250 hectares. Plus au nord, les réservoirs de kérosène alimentant la plateforme aéroportuaire occupent une quinzaine d'hectares sur la commune de Chennevières-lèsLouvres, ce qui limite également l'extension de ce village pour cause de risque élevé d'accident technologique (classement Seveso II).

31 Les rues des villages concernés offrent visuellement, au premier abord, une impression de crise. La plupart des maisons sont anciennes et leur entretien apparaît souvent déficient. La situation est particulièrement frappante au "vieux pays» de Goussainville, où de nombreuses demeures ont été murées par les Aéroports de Paris (ADP), qui proposait, dans les années 1970 et 1980, le rachat à l'amiable des bâtiments, dont la destruction est cependant interdite par le classement de l'église aux monuments historiques (Millot et al., 2002).

32 À l'inverse des villages du nord-ouest du Pays de France, concernés par l'influence cantilienne, ceux-ci ne connaissent pas de valorisation résidentielle, récréative et environnementale claire, en dépit de leur caractère rural et de leur grande proximité urbaine. Ils sont, au contraire, investis par des familles aux revenus modestes, pour qui 
la dépréciation de l'immobilier associée aux nuisances constitue une opportunité d'accéder à un logement plus grand à moindre coût (Faburel, 2003).

Figure 4. L'influence des activités de la plateforme de Roissy : diffusion et rupture

Plate-forme aéroportuaire

Unité urbaine de Paris

I. Couloir de bruit et rupture urbaine

Zone de nuisances

sonores majeures

Villages "sacrifiés"

par les nuisances

Autres équipements

porteurs de nuisances :

Centre d'enfouissement des déchets

뜰 Poste électrique

- Réservoirs de kérosène

II. Un développement diffus vers le nord de la plate-forme

Zones d'activités logistiques développées depuis 2000 Villes du Pays de France (quartiers résidentiels)

6ros villages périurbains

Zones d'activités préexistantes

Sources: IGN, Maison de IEnvironnement, enquêtes personnelles.

J.-B. GRISON, septembre 2011

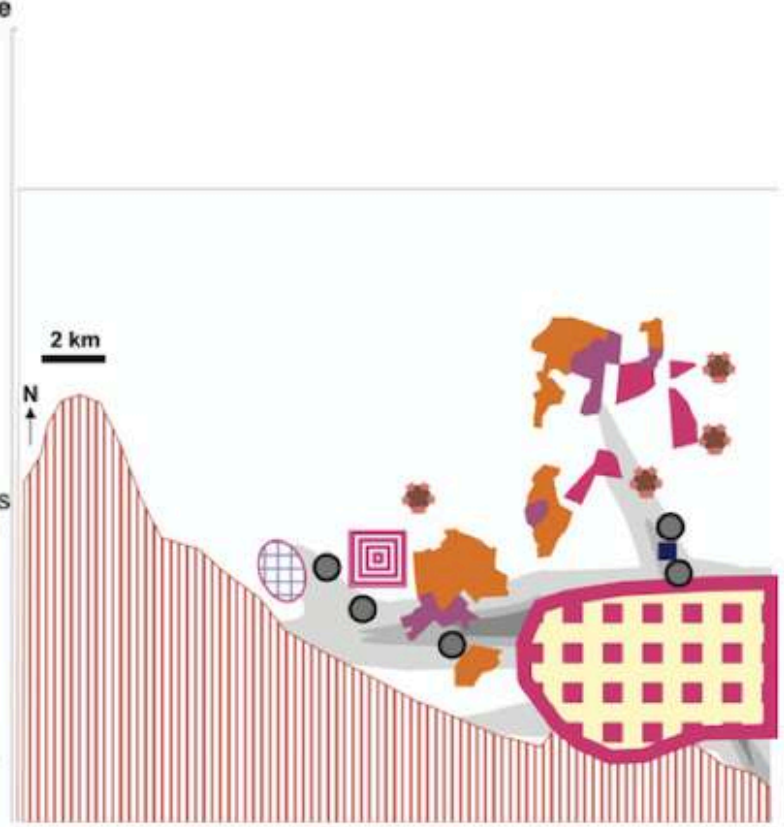

\section{Un développement très important des zones d'activités, de plus en plus orienté vers l'espace périurbain}

Depuis l'ouverture de la plateforme aéroportuaire, de nombreuses zones d'activités se sont développées, tout particulièrement dans le domaine de la logistique et des transports (Berthon, 2003). Dans un premier temps, ces activités sont restées concentrées à proximité immédiate de Roissy, principalement au sud-ouest (zones d'activités Parinor 1 et 2). Plus récemment, un développement resté jusque-là timide s'est amplifié en direction des zones périurbaines du Pays de France. L'exemple de Vémars et Saint-Witz est caractéristique. Dans le courant des années 2000, de vastes zones d'activités, essentiellement constituées de plateformes logistiques, s'y sont développées. Plusieurs centaines d'hectares sont ainsi concernées, ce qui marque profondément le paysage, où l'agriculture recule rapidement. Plus récemment encore (depuis 2010), ce développement se poursuit entre Louvres et Villeron.

Spatialement, l'implantation de ces activités répond plutôt à une logique de diffusion interstitielle: leur localisation n'est pas continue, ni contiguë à la plateforme aéroportuaire, mais occupe les zones restées à l'écart, tant du périmètre des plus fortes nuisances sonores, que des secteurs soumis à des dispositifs de protection ou à des contraintes urbanistiques trop fortes. 


\section{Conclusion}

"quantitatif », c'est-à-dire associé à une forte extension de l'espace résidentiel (mais aussi, et surtout dans le cadre de l'influence de Roissy, de zones d'activités), tantôt de «qualitatif », lorsque les villages ne s'étendent pas, mais que les liens avec la ville, par les activités présentes comme par la composition sociologique de la population, s'accroissent. La volonté politique consistant à refuser l'extension urbaine et à protéger l'espace non bâti, dans une perspective de valorisation paysagère, peut alors être qualifiée d'inertie dynamique. À l'inverse, l'inertie des villages dépréciés par les équipements répulsifs et les nuisances sonores peut être qualifiée d'inertie structurelle ou fonctionnelle. Les documents d'urbanisme et les volontés politiques locales jouent un rôle important dans ces évolutions. La totalité des communes sont pourvues d'un plan d'occupation des sols (POS) ou d'un plan local d'urbanisme (PLU), et la majorité d'entre elles a approuvé un nouveau PLU dans le courant des années 2000. Dans les parties nord et ouest, davantage concernées par l'influence cantilienne, les révisions ont entraîné, dans plusieurs cas, une limitation des ambitions d'extension des zones urbaines. Cela se vérifie notamment pour les communes de Fosses, Bellefontaine, Puiseux-en-France, où l'on constate par exemple que les prévisions d'urbanisation du schéma directeur de 1995 ont été largement revues à la baisse. Le classement du site de la vallée de l'Ysieux au titre de la loi de 1930 est en partie responsable de cette réorientation. À l'inverse, les gros bourgs ou petites villes plus proches de la plateforme aéroportuaire de Roissy, mais hors de la zone de bruit (Louvres, Villeron, Vémars), présentent des objectifs de développement plus ambitieux.

Pour toutes ces raisons, et malgré un encadrement urbain puissant et multidirectionnel, il est vraisemblable que les poches d'espace rural du Pays de France ne disparaissent pas. En revanche, sa partie déjà urbaine (chapelet de petites villes le long de la ligne de chemin de fer, ainsi que les gros bourgs, désormais urbains, de Vémars et Saint-Witz) devrait se renforcer et atteindre, à long terme, une quasicontinuité urbaine. En somme, le cœur du Pays de France est typiquement dans le cas d'une vocation périurbaine persistante: déjà ancienne, cette dernière continue à conjuguer un dynamisme très fort avec une certaine stabilité. 


\section{BIBLIOGRAPHIE}

Bastié, J., Géographie du Grand Paris, Paris, Masson, 1984, 208 p.

Berger, M., Les Périurbains de Paris : de la ville dense à la métropole éclatée ?, Paris, CNRS éditions, coll. «Espaces et Milieux», 2004, 320 p.

Berthon, E., « Roissy, le dynamisme du pôle de développement dans un environnement social en difficulté », Cahiers de l'Institut d'aménagement et d'urbanisme de la Région Île-de-France, Paris, Iaurif, 2003, no 139-140, p. 82-87.

Cavard, J.-C., « Pays de France et Plaine de France : réalités géographiques d'une banlieue parisienne », Cahiers du Crepif, Paris, Crepif, 1994, n 46, p. 139-169.

Clozier, R., La Gare du nord, Paris, J.-B. Baillière, 1940, 294 p.

Faburel, G., « Autour de Roissy et d'Orly : une géographie singulière ? », Cahiers de l'Institut d'aménagement et d'urbanisme de la Région Île-de-France, Paris, Iaurif, 2003, n 139-140, p. 98-99.

Faburel, G. et Barraque, B., « Les impacts territoriaux du bruit des avions. Le cas de l'urbanisation à proximité de Roissy CDG. Ne pas évaluer pour pouvoir tout dire, et son contraire ", rapport final du Centre de recherche sur l'espace, les transports, l'environnement et les institutions locales (Creteil), pour l'ADEME, dans le cadre du programme « Concertation, décision et environnement » du ministère de l'Aménagement du territoire et de l'Environnement, mars, 2002, $43 \mathrm{p}$.

Grison, J.-B., « La très petite commune en France : héritage sans avenir ou modèle original ? » thèse de géographie, Clermont-Ferrand, université Blaise-Pascal, 2009, 406 p.

Grison, J.-B., «Stratégies municipales et logiques de protection de l'espace en milieu périurbain : le cas de l'aire urbaine de Paris », dans Molinero Hernando, F. (sous la dir. de), Espacios naturales protegidos, actes du colloque hispano-français de géographie rurale, Baeza, AGE/Universidad Internacional de Andalucia, 2008, p. 373-384.

Millot et al., Naissance d'une banlieue, mort d'un village : Goussainville, 2002, conception : Olivier Millot, Pierre Gaudin ; réalisation : Sidney Jézéquel ; production : Les films Roger Leenhardt ; $\mathrm{CNC}$, Images de la Culture, 52 minutes.

Nadler, G., « Aéroport de Roissy 1974-2004 : mutations vers un nouvel urbanisme », mémoire de troisième cycle DPLG, Paris, École nationale supérieure d'architecture Paris-Malaquais, 2004, $175 \mathrm{p}$.

Plet, F., « Banlieue des banlieues : pouvoirs et politique dans l'espace périurbain au nord de Paris ", Hérodote, Paris, Masson, 1986, n 43, p. 104-122.

Poulot, M. et Rouyres, T., « Vivre dans des espaces de faible densité aux portes d'une grande agglomération : l'exemple des campagnes franciliennes », Habiter et Vivre dans les campagnes de faible densité, Clermont-Ferrand, Ceramac, Presses universitaires Blaise Pascal, 2007, p. 215-232.

\section{NOTES}

1. Le Pays de France n'est pas une entité politique définie. La zone étudiée correspond approximativement aux 
deux communautés de communes de Roissy Porte de France et Cœur du Pays de France ; une partie appartient au Parc naturel régional Oise-Pays de France, une autre au Scot de l'est du Vald'Oise.

2. Institut national de la statistique et des études économiques.

\section{RÉSUMÉS}

Le Pays de France est une poche d'espace périurbain située entre le nord de l'agglomération parisienne, la zone aéroportuaire de Roissy et l'agglomération de Chantilly. Le développement de cet espace est lié aux influences différenciées de ces trois entités urbaines, dont les composantes morphologiques et fonctionnelles divergent. Ces influences font du Pays de France une «zone tampon ", dont les paysages, travaillés par les éléments urbains externes, donnent à chaque localité un aspect singulier. Le front d'urbanisation à dominante populaire se conjugue ainsi diversement avec les activités de loisirs et la forte valorisation résidentielle propres à Chantilly, tandis que la diffusion des activités liées à l'aéroport de Roissy, mais aussi les zones de nuisances sonores imposent d'autres clivages territoriaux. En définitive, on peut opposer, dans les formes de dynamiques spatiales à l'œuvre dans le Pays de France, des logiques radiales (agglomération parisienne), frontales (aire cantilienne), et de diffusion ou fracture (Roissy).

The "Pays de France" is an outer-urban territory, in the north of the Parisian suburbs, south of Chantilly town, and west of Roissy airport. This three urban spaces influence the periurban development, with different sorts of dynamics. The "Pays de France" is so a "buffer strip", where each locality knows its own landscape changes. The growth of Parisian suburbs follow some major trends, threw a development of low coast residential areas. From the north, the influence of Chantilly city ("French horse capital") progress as a front of up-market residential properties and spare-time activities. The airport zone, which represents a big employment hub, has a double influence on the "Pays de France" : some localities are growing with big activities areas; other are "sacrificed" because of too high noise pollution. Finally, we can see several spatial trends : radial, frontal, diffusion or divide logics.

\section{INDEX}

Keywords : parisian suburbs, social-spatial divides, urban and rural morphology, territorial dynamics, city-countryside relationships

Mots-clés : aire urbaine de Paris, clivages sociospatiaux, morphologie urbaine et rurale, dynamique territoriale, pression urbaine

\section{AUTEUR}

\section{JEAN-BAPTISTE GRISON}

Géographe, il est chercheur associé au Ceramac, université Blaise-Pascal, Clermont-Ferrand. jbgrison1[at]gmail[dot]com 